Enferm Bras 2019;18(3):398-405

https://doi.org/10.33233/eb.v18i3.2508

\title{
ARTIGO ORIGINAL \\ Autocuidado em hanseníase: comportamento de usuários atendidos na rede de atenção primária à saúde
}

Paula Soares Carvalho*, Karen Krystine Gonçalves de Brito, D.Sc.* ${ }^{*}$, Emanuelle Malzac Freire de Santana ${ }^{* * *}$, Siméia Macêdo de Lima ${ }^{* * *}$, Smalyanna Sgren da Costa Andrade, D.Sc. ${ }^{* * * *}$, Matheus de Medeiros Nóbrega ${ }^{\star \star \star \star \star *}$, Maria Júlia Guimarães Oliveira Soares, D.Sc. ${ }^{* \star \star \star \star \star *}$

*Mestranda em Enfermagem pela Universidade Federal da Paraíba, João Pessoa/PB, **Enfermeira do ambulatório de feridas da Secretaria Municipal de Saúde de Bayeux, Paraíba, ***Doutoranda em Enfermagem pela Universidade Federal da Paraíba, Docente do curso de Fisioterapia das Faculdades Nova Esperança, João Pessoa/PB, ${ }^{* * *}$ Graduação em Enfermagem pela Universidade Federal da Paraíba, João Pessoa/PB, ${ }^{* * * * *}$ Docente do curso de Enfermagem das Faculdades Nova Esperança, João Pessoa PB, ${ }^{* \star * \star * *}$ Mestrando pelo programa de pós

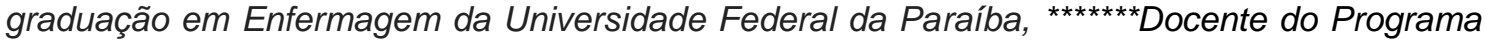
de Pós-Graduação em Enfermagem da Universidade Federal da Paraíba, João Pessoa/PB

Recebido em 10 de setembro de 2018; aceito em 22 de abril de 2019.

Correspondência: Emanuelle Malzac Freire de Santana, Av. Julieta Marinho Marsciano, 109/204, 58035-000 João Pessoa PB, E-mail: manumalzac@gmail.com; Paula Soares Carvalho: paaula-soares@live.com; Karen Krystine Gonçalves de Brito: karen_enf@yahoo.com.br; Emanuelle Malzac Freire de Santana: manumalzac@gmail.com; Siméia Macêdo de Lima: simeiamacedo@windowslive.com; Smalyanna Sgren da Costa Andrade: nana_sgren@hotmail.com; Matheus de Medeiros Nóbrega: matheusnobrega0@gmail.com; Maria Júlia Guimarães Oliveira Soares: mmjulieg@gmail.com

\section{Resumo}

Introdução: A hanseníase é uma doença negligenciada e incapacitante de relevância para a saúde pública brasileira. $\mathrm{O}$ autocuidado se configura como estratégia de prevenção contra suas incapacidades físicas. Objetivo: Investigar as competências dos indivíduos com hanseníase atendidos na atenção primária à saúde, direcionado a ações de autocuidado através da escala Appraisal of Self-care Agency. Métodos: Pesquisa quantitativa realizada nas unidades de saúde da família em João Pessoa/PB, com 22 participantes de janeiro a março de 2017. Utilizou-se na coleta de dados um instrumento sociodemográfico e a escala Appraisal of Self-care Agency. Os dados foram analisados por técnicas de estatística descritiva e inferencial através dos testes $U$ de Mann-Whitney e Kruskal Wallis. Resultados: Perfil caracterizado por maioria de mulheres, com média de 52 anos, tipo multibacilar, grau de incapacidade não especificado, com média de 92 pontos na escala ASA. Conclusão: Observou-se em uma taxa majoritária dos entrevistados a competência para o autocuidado.

Palavras-chave: hanseníase, atenção primária à saúde, autocuidado, Enfermagem.

\begin{abstract}
Self-handled in leprosy: behavior of users attended in the primary health care network Introduction: Leprosy is a neglected and disabling disease of relevance to Brazilian Public Health. Self-care is configured as a prevention strategy against the physical disabilities. Objective: To investigate the skills of individuals with leprosy treated in primary health care, directed to self-care actions through the Appraisal of Self-care Agency Scale. Methods: Quantitative research carried out at the family health units in João Pessoa, Paraíba, Brazil, with 22 participants from January to March 2017. A sociodemographic instrument and the Appraisal of Self-care Agency scale were used in the data collection. Data were analyzed by descriptive and inferential statistics using the Mann-Whitney and Kruskal Wallis U tests. Results: Profile characterized by majority of women, with a mean of 52 years old, multibacillary type, degree of disability not specified, with a mean of 92 points on the ASA scale. Conclusion: Most of respondents had the competence to self-care.
\end{abstract}

Key-words: leprosy, primary health care, selfcare, Nursing. 


\section{Resumen \\ Autocuidado en la lepra: comportamiento de usuarios atendidos en la red de atención primaria a la salud}

Introducción: La lepra es una enfermedad negligente e incapacitante de relevancia para la salud pública brasileña. El autocuidado se configura como estrategia de prevención contra sus incapacidades físicas. Objetivo: Investigar las competencias de los individuos con hanseniasis atendidos en la atención primaria a la salud, dirigido a acciones de autocuidado a través de la escala de evaluación de la autoestima. Métodos: Investigación cuantitativa realizada en las unidades de salud de la familia en João Pessoa, Paraíba, Brasil, con 22 participantes de enero a marzo de 2017. Se utilizó en la recolección de datos un instrumento sociodemográfico y la escala Appraisal of Self-care Agency. Los datos fueron analizados por técnicas de estadística descriptiva e inferencial a través de las pruebas U de Mann-Whitney y Kruskal Wallis. Resultados: Perfil caracterizado por mayoría de mujeres, con media de 52 años, tipo multibacilar, grado de incapacidad no especificado, con promedio de 92 puntos en la escala ASA. Conclusión: Se observó en la mayoría de los entrevistados la competencia para el autocuidado.

Palabras-clave: lepra, atención primaria de salud, autocuidado, Enfermería.

Introdução

A hanseníase é uma doença crônica, infectocontagiosa, negligenciada e que apresenta distribuição mundial, colocando o Brasil como o segundo país mais endêmico. Apesar de curável, pode causar aos indivíduos incapacidades físicas e consequências biopsicossociais devido ao acometimento dermatoneurológico ocasionado pelo Mycobacterium leprae, configurando-se como problema de saúde pública [1,2].

O diagnóstico e tratamento tardios trazem consigo grandes consequências como as incapacidades físicas e deformidades, convidando o indivíduo acometido a vivenciar de maneira particular o estigma e preconceito da doença. As incapacidades podem acarretar restrições na realização de atividades diárias, limitações da vida social e prejuízos psicológicos [3].

No ano de 2017 foram registrados globalmente 210.671 novos casos de hanseníase. Desde a última década, o Brasil vem apresentando uma tendência de diminuição dos casos novos, entretanto, essa realidade tem se transformado, obtendo-se um aumento de números de casos entre o ano de 2017 e 2018, perfazendo 27.884 casos nesse último ano [1]. Desses, a região nordeste apresenta-se com maior endemicidade, sendo responsável por aproximadamente 11.420 casos novos [4].

Integrante da região Nordeste, o estado da Paraíba tem se mostrado ativo no combate a hanseníase, tendo registrado no ano de 2018, 510 casos novos, e sua capital, João Pessoa, 233 casos novos, alcançando no ano de 2016 a meta de possuir menos de 1 caso/10.000 habitantes [4].

Cerca de $25 \%$ das pessoas com hanseníase manifestam alguma incapacidade. No Brasil, aproximadamente $23,3 \%$ dos casos registrados anualmente apresentam graus de incapacidade física (GIF) I e II, fazendo-se importante a realização do acompanhamento destes por parte dos profissionais de saúde [5]. Acredita-se que não exista outra doença que produza tantas incapacidades físicas, emocionais e sociais como a hanseníase [6].

No âmbito da prevenção e controle de incapacidades, o autocuidado se estabelece como uma das principais estratégias. O mesmo pode ser definido como a realização de atividades cotidianas que atuam em prol da manutenção de sua vida, saúde e bem-estar, podendo ser influenciado por habilidades, limitações, condições culturais, sociais e econômicas que interferem de modo direto na saúde de quem o realiza [7]. As demandas de autocuidado estão diretamente relacionadas aos olhos, face, membros superiores e inferiores, considerando-se que são os principais sítios de acometimentos cutâneo e neural [8].

$\mathrm{Na}$ perspectiva de relacionar às funções humanas essenciais para a manutenção da saúde e do bem-estar dos indivíduos, Dorothea Elizabeth Orem, propôs que os indivíduos, quando capazes, devem cuidar de si mesmos. Em face do surgimento da impossibilidade de realizar estas atividades básicas, torna-se necessário a intervenção do cuidado através de um terceiro, tornando assim o ser que não exerce a prática, dependente de outrem [9].

Em se tratando da hanseníase, as práticas de cuidado para consigo faz parte da abordagem de manutenção do tratamento, a partir do momento em que o enfermeiro juntamente 
com o paciente é capaz de identificar as condições e atividades necessárias para desenvolvêlas de forma positiva [9].

A adesão ao autocuidado depende de diversos fatores, posto que se trata de medidas cotidianas que devem ser efetuadas por toda vida. Acredita-se que o indivíduo acometido pela hanseníase além de ser afetado fisicamente pela doença, lida com o conflito diário do paradigma que é cuidar-se e relacionar-se com as diversas ações de cuidar de si mesmo [10].

Neste contexto, a Estratégia Saúde da Família possui relevante importância no cumprimento do papel da promoção, prevenção e controle de agravos na saúde pública, devendo a hanseníase ser tratada de forma prioritária através da poliquimioterapia (PQT) disponível no Sistema Único de Saúde. Além disso, ações de educação em saúde e avaliação das capacidades dos utentes são atividades válidas e essenciais para instrução e realização do autocuidado [11].

Em face ao exposto, é fundamental avaliar as competências dos utentes ao tratamento para viabilizar a promoção de estratégias e ações educativas direcionadas e, portanto, mais efetivas. Nesta perspectiva, o objetivo deste estudo é investigar as competências dos pacientes com hanseníase, atendidos na atenção primária à saúde, direcionado a ações de autocuidado.

Material e métodos

Trata-se de estudo quantitativo, que foi desenvolvido com pacientes acometidos pela hanseníase e atendidos nas Unidades de Saúde da Família (USF) da rede de atenção primária a saúde, localizadas no município de João Pessoa, Paraíba, Brasil.

A atenção básica deve ser responsável pela resolução de aproximadamente $80 \%$ de todos os problemas de saúde da população, desempenhando papel importante nas políticas direcionadas à prevenção de agravos e à promoção da saúde [12]. No município de João Pessoa, as USF estão distribuídas em cinco distritos sanitários, a saber:

- Distrito I: Cruz das Armas, Jaguaribe, Costa e Silva, Alto do Mateus, Indústrias, Oitizeiro, Novais, Jardim Veneza e Ernani Sátiro.

- Distrito II: Geisel, Cristo, Varjão, Água Fria, João Paulo II, Cuiá, Cidade dos

Funcionários, Grotão, Ernesto Geisel e Gramame.

- Distrito III: Mangabeira, Valentina, Jardim Cidade Universitária, Jardim São Paulo, Bancários, Planalto da Boa Esperança, Cidade dos Colibris, Paratibe, José Américo e Gramame.

- Distrito IV: Torre, Róger, Tambiá, Centro, Mandacaru, Alto do Céu, Varadouro, Ipês, Trincheiras, llha do Bispo e Treze de maio.

- Distrito V: Miramar, Tambauzinho, Manaíra, Torre, Tambaú, Altiplano Cabo Branco, Bessa, Castelo Branco, Portal do Sol, Expedicionários e Penha.

A população do estudo foi constituída por indivíduos acometidos pela hanseníase que atendessem aos seguintes critérios de inclusão: idade maior a 18 anos; em tratamento a partir da $2^{2}$ dose de PQT, e em um dos cenários da pesquisa; e apresentar capacidade cognitiva preservada (autorreferida ou percebida pela pesquisadora). Foram utilizados como critérios de exclusão: casos novos da doença, tendo em vista que estes podem apresentar alguma das fases do luto e não aceitação da sua condição de saúde; pacientes em tratamento apenas de reação hansênicas e em alta por cura/abandono/transferência.

Assim, após realizada busca ativa em todos os distritos sanitários do município, foram constatados 44 indivíduos cadastrados em tratamento para doenças. Desses, 03 eram menores de idade, 03 haviam descontinuado o tratamento por abandono, 01 já havia comparecido ao serviço para receber a última dose supervisionada e, por isso, não retornaria, 03 não aceitaram participar da pesquisa.

Dos 34 pacientes selecionados, 12 não foram contactados pela inviabilidade de acesso pelo contexto socioespacial em que habitavam, sendo estas áreas de difícil acesso, violência urbana e vulnerabilidade à pesquisadora. Assim, foram entrevistados e compuseram a amostra 22 indivíduos.

A coleta de dados foi realizada entre os meses de janeiro a março de 2017, através de dois instrumentos estruturados. O primeiro contendo dados sociodemográficos (sexo, idade, nível de escolaridade, estado civil, renda familiar média, religião), e clínico-epidemiológicos (dose do tratamento, classificação operacional, status de baciloscopia, forma clínica, grau de 
incapacidade física no diagnóstico, comprometimento neural, presença de doenças associadas, ocorrência de reações hansênicas, presença de úlceras palmares e plantares e neurite).

O segundo instrumento utilizado foi a escala Appraisal of Self-care Agency - ASA (Avaliação da competência para o autocuidado) com o intuito de mensurar a capacidade do indivíduo para engajar-se em intervenções essenciais ao seu cuidado. A escala foi desenvolvida por Isenberg, Evers e Philipsen em 1987, baseado na Teoria de Enfermagem de Dorothea Orem, sendo seu conteúdo validado pela mesma e outras 8 doutoras, com especialidade no conceito que diz respeito ao autocuidado. A escala é exposta em dois formatos, ASA-A_Autoavaliação e ASA-A_Avaliação por outros, sendo a primeira adaptada e validada para cultura brasileira e denominada Escala para avaliar a capacidade de autocuidado [13].

A ASA-A é composta por 24 itens com atribuições de valores que variam entre 1 e 5 , associada com uma escala Likert na seguinte disposição: 1 (discordo totalmente), 2 (discordo), 3 (nem concordo nem discordo), 4 (concordo) e 5 (concordo totalmente). As respostas têm valor mínimo de 24 pontos e máximo de 120 , sendo a pontuação máxima relacionada à melhor capacidade [13].

Os dados obtidos foram analisados através de técnicas de estatística descritiva e inferencial. Foram tabulados e codificados no Microsoft Office Excel 2016 para posterior análise no software Statistical Package for the Social Sciences (SPSS) versão 21.0 utilizando os testes U de Mann-Whitney e Kruskal Wallis.

O projeto foi aprovado no Comitê de Ética e Pesquisa (CEP) do Centro de Ciências em Saúde da Universidade Federal da Paraíba, sob o protocolo n¹.824.702 e de acordo com o Certificado de Apresentação para Apreciação Ética de número 59488616.2.0000.5188. Ressaltase que foram obedecidas todas as observâncias éticas contempladas na Resolução n 466/12 do Conselho Nacional de Saúde.

Resultados

Os dados compilados apontam predominância dos entrevistados com idade variando entre 21 e 84 anos com média de 52 anos, sexo feminino (54,5\%), estado civil (54,5\%), religião católica $(63,6 \%)$. Quanto à escolaridade e renda, $40,9 \%$ referiram possuir o ensino médio completo e $63,6 \%$ renda menor ou igual a um salário mínimo.

Clinicamente sobressaíram-se os casos multibacilares (81,8\%), baciloscopia positiva (50\%), forma clínica dimorfa $(40,9 \%)$. Aproximadamente $40 \%$ dos indivíduos tiveram classificação do GIF desconhecida/não registrada em seus prontuários, e em sua maioria não apresentaram comprometimento neural em face $(68,2 \%)$, membros superiores $(59,1 \%)$ e membros inferiores (50\%). Quanto às reações hansênica, $72,7 \%$ relataram a ausência de episódio e $77,3 \%$ não referiram neurite.

Para os resultados da escala, a Tabela I apresenta a sua distribuição de acordo com as categorias. O valor mínimo obtido foi de 68 pontos (categoria regular) e o máximo de 114 pontos (ótima), com média de 92 pontos ( $\mathrm{DP} \pm 13,37$ ) (categoria muito boa).

Tabela I - Distribuição quantitativa das categorias de pontuação da Escala Appraisal of self-Care Agency, de acordo com frequência e percentual. João Pessoa, PB, 2017. (N=22)

\begin{tabular}{lll}
\hline Categoria & Frequência (n) & Percentual (\%) \\
\hline Ruim & - & - \\
Regular & 2 & 9,1 \\
Boa & 7 & 31,8 \\
Muito boa & 9 & 40,9 \\
Otima & 4 & 18,2 \\
\hline
\end{tabular}

Fonte: Dados da pesquisa, 2017.

As tabelas II e III exibem a associação entre a classificação da escala ASA-A e as variáveis sociodemográficas e clínico-epidemiológicas, sendo observada relevante associação apenas entre a variável acometimento da face e classificação operacional com $p$-valor $\leq 0,05$. 
Tabela II - Distribuição quantitativa cruzada, relacionando as características sociodemográficas e categorias de pontuação da escala ASA-A. João Pessoa/ PB, 2017. ( $N=22)$.

\begin{tabular}{|c|c|c|c|c|c|c|}
\hline \multirow[t]{2}{*}{ Variáveis } & & \multicolumn{4}{|c|}{ Classificação ASA-A } & \multirow[t]{2}{*}{ P-valor } \\
\hline & & regular & boa & muito boa & ótima & \\
\hline Sexo & Masculino & $2(20 \%)$ & $2(20 \%)$ & $4(40 \%)$ & $2(20 \%)$ & $0,834^{\star *}$ \\
\hline \multirow[t]{5}{*}{ Escolaridade } & $\begin{array}{l}\text { Sem } \\
\text { escolaridade }\end{array}$ & - & - & $1(50 \%)$ & $1(50 \%)$ & $0,274^{\star \star \star}$ \\
\hline & Alfabetizado & $1(50 \%)$ & $1(50 \%)$ & - & - & \\
\hline & Ens. fund. & - & $3(37,5 \%)$ & $4(50,0 \%)$ & $1(12,5 \%)$ & \\
\hline & Ens. médio & $1(11,1 \%)$ & $3(33,3 \%)$ & $3(33,3 \%)$ & $2(22,2 \%)$ & \\
\hline & Ensino Sup. & - & - & $1(100 \%)$ & - & \\
\hline \multirow[t]{3}{*}{ Renda } & $\leq 1$ salário & $1(7,1 \%)$ & $6(42,9 \%)$ & $4(28,6 \%)$ & $3(21,4 \%)$ & $0,869^{* * *}$ \\
\hline & >1-3 salários & $1(14,3 \%)$ & $1(14,3 \%)$ & $4(57,1 \%)$ & $1(14,3 \%)$ & \\
\hline & $>3-5$ salários & - & - & $1(100 \%)$ & - & \\
\hline \multirow[t]{4}{*}{ Religião } & Católica & $1(7,1 \%)$ & $4(28,6 \%)$ & $5(35,7 \%)$ & $4(28,6 \%)$ & $0,748^{* * *}$ \\
\hline & Evangélica & $1(33,3 \%)$ & - & $2(66,7 \%)$ & - & \\
\hline & Outras & - & - & $1(100 \%)$ & - & \\
\hline & Ignorado* & - & $3(75,0 \%)$ & $1(25,0 \%)$ & - & \\
\hline
\end{tabular}

* Os dados não obtidos/ignorados não foram contabilizados para o p-valor; ${ }^{* *}$ Teste de Mann Whitney; ${ }^{* * *}$ Teste de Kruskal Wallis. Fonte: Dados da pesquisa, 2017.

Tabela III - Distribuição quantitativa cruzada, relacionando as características clínicas e categorias de pontuação da escala ASA-A. João Pessoa/PB, 2017(N=22).

\begin{tabular}{|c|c|c|c|c|c|c|}
\hline \multirow[t]{2}{*}{ Variáveis } & & \multicolumn{3}{|c|}{ Classificação ASA-A } & & \multirow[t]{2}{*}{ P-valor } \\
\hline & & regular & boa & muito boa & ótima & \\
\hline \multirow[t]{3}{*}{ GIF } & GIF 0 & - & $1(20,0 \%)$ & $3(60,0 \%)$ & $1(20,0 \%)$ & $0,641^{\star \star *}$ \\
\hline & GIF 1 & $2(25,0 \%)$ & $1(12,5 \%)$ & $3(37,5 \%)$ & $2(25,0 \%)$ & \\
\hline & Ignorado* & - & $5(55,6 \%)$ & $3(33,3 \%)$ & $1(11,1 \%)$ & \\
\hline \multirow{3}{*}{$\begin{array}{l}\text { Acometimento } \\
\text { de Face }\end{array}$} & Sim & $1(50 \%)$ & $1(50 \%)$ & - & - & $0,056^{\#}$ \\
\hline & Não & $1(6,7 \%)$ & $3(20,0 \%)$ & $8(53,3 \%)$ & $3(20,0 \%)$ & \\
\hline & Ignorado* & - & $3(60,0 \%)$ & $1(20,0 \%)$ & $1(20,0 \%)$ & \\
\hline \multirow{3}{*}{$\begin{array}{l}\text { Acometimento } \\
\text { de MMSS }\end{array}$} & Sim & $1(20,0 \%)$ & $1(20,0 \%)$ & $3(60,0 \%)$ & - & $0,366^{\star *}$ \\
\hline & Não & $1(7,7 \%)$ & $3(23,1 \%)$ & $6(46,2 \%)$ & $3(23,1 \%)$ & \\
\hline & Ignorado* & - & $3(75,0 \%)$ & - & $1(25,0 \%)$ & \\
\hline \multirow{3}{*}{$\begin{array}{l}\text { Acometimento } \\
\text { de MMII }\end{array}$} & Sim & $1(14,3 \%)$ & $2(28,6 \%)$ & $4(57,1 \%)$ & - & $0,241^{* *}$ \\
\hline & Não & $1(9,1 \%)$ & $2(18,2 \%)$ & $5(45,5 \%)$ & $3(27,3 \%)$ & \\
\hline & Ignorado* & - & $3(75,0 \%)$ & - & $1(25,0 \%)$ & \\
\hline \multirow{3}{*}{$\begin{array}{l}\text { Clas. } \\
\text { Operacional }\end{array}$} & Paucibacilar & - & $3(100 \%)$ & - & - & $0,054^{\#}$ \\
\hline & Multibacilar & $1(5,6 \%)$ & $4(22,2 \%)$ & $9(50,0 \%)$ & $4(22,2 \%)$ & \\
\hline & Ignorado* & 1 (100\%) & - & - & - & \\
\hline
\end{tabular}

Fonte: Dados da pesquisa, 2017; *Os dados não obtidos/ignorados não foram contabilizados para o p-valor; \#P-valor significativo estatisticamente; ${ }^{* \star}$ Teste de Mann Whitney; ${ }^{* \star *}$ Teste de Kruskal Wallis.

Os resultados apontam amostra majoritariamente do sexo feminino, com ensino médio incompleto e renda menor ou igual a um salário mínimo. Quanto à classificação na Escala ASAA $72,7 \%$ apresentaram capacidade para se autocuidar entre boa $(31,8 \%)$ e muito boa $(40,9 \%)$. Quanto às associações das características sociodemográficas e clínicas com as classificações, apenas o acometimento facial e classificação operacional, tiveram valores estatisticamente significativos $(p \leq 0,05)$.

Discussão

A hanseníase continua sendo considerada um dos grandes problemas de saúde pública do Brasil, que apesar de ser uma enfermidade curável e tratável, sua alta prevalência se dá por consequência de uma evolução lenta, silenciosa, com rápida facilidade de proliferação de focos de infecções, atingindo alto comprometimento de incapacidades física no acometido. Quanto 
mais tardio o diagnóstico, maior a chance de acarretar grandes complicações e sequelas, comprometendo a qualidade de vida daqueles que possui a doença [14].

Partindo-se desse pressuposto, acredita-se que a realização de ações de autocuidado seja de fundamental importância para minimizar ou prevenir consequências, como incapacidades para atividades laborais do cotidiano e deformidades físicas, e auxiliar o indivíduo no reconhecimento de sua dor, de suas angústias, esperanças e perspectivas [10].

Nesse contexto preponderante os achados desta amostra são de grande relevância, pois apontam a presença de relações atitudinais favoráveis a capacidade de autocuidado dos indivíduos. Quando isso é propiciado pela atenção primária à saúde, há ainda melhores prognósticos, uma vez que neste nível de atenção devem ser intensificadas ações relacionadas à prevenção de agravos e a promoção à saúde [15], contribuindo para uma melhor qualidade de vida dos indivíduos.

Visando a prevenção de incapacidades por meio da prática do autocuidado, infere-se a importância de implementação de estratégias que busquem avaliar a capacidade que o indivíduo tem de realizar o cuidado do seu próprio corpo. $\mathrm{O}$ autocuidado configura-se como ferramenta principal para a prevenção e controle dos agravos. Os profissionais de saúde atuam junto ao cliente para proporcionar medidas educativas que incentivam a adesão e realização do autocuidado continuamente. Nesse contexto, a avaliação da capacidade para tais atividades faz com que o profissional possa traçar métodos educativos para auxiliar o paciente dentro das limitações e individualidades de cada ser [16].

Consubstancia-se, portanto a inovação deste estudo ao buscar esclarecer arestas tão pouco evidenciadas na literatura, muito embora a capacidade de autocuidado esteja intrinsecamente relacionada com a adesão ou não do indivíduo a prática. A afirmativa se confirma ao observar a associação estatisticamente significativa entre a capacidade para autocuidado (avaliada pela escala ASA-A) e as características clínicas da amostra.

Quanto à associação da ASA-A com o acometimento facial pode-se hipotetizar que por ser um local de grande visibilidade e graves formas de deformidades (logoftalmo, triquíase, perda de cílios, entre outros), os participantes apresentam a tendência e a capacidade de prover ações de autocuidado com essa área especificamente.

É válido enaltecer a necessidade de avaliar de forma cautelosa e criteriosa este sítio corporal. Quando alojadas, as incapacidades poderão evoluir para deformidades trazendo consigo as dificuldades de realização de atividades diárias, perda de identidade restringindo ainda mais o convívio social [16].

Outro estudo enfatiza ainda que as alterações na estrutura e função corporais acarretadas pela hanseníase levam os pacientes a apresentarem mudanças na percepção da própria imagem corporal e se tratando principalmente da face, há relatos responsáveis por mudanças de concepção da própria identidade daqueles indivíduos [10].

Outro dado a ser destacado neste estudo concerne à associação significativa entre a escala ASA-A e a classificação operacional para hanseníase. Evidencia-se que os indivíduos com maior carga bacilar estão mais propensos a se autocuidar ao passo que foram classificados com maior grau de capacidade conforme escala avaliativa.

O acometimento pelas formas multibacilares da doença, aumenta em nove vezes a chance de desenvolver algum grau de incapacidade física. Tendo isso como referência, acreditase que há nesse caso uma consciência de risco que justifique esse valor significativo pelos entrevistados [17].

Estudo aponta que a maior frequência da forma clínica existente da hanseníase são as multibacilares, e é neste grupo que ocorre a maioria das deformidades e incapacidades visíveis na hanseníase, sendo considerada a mais grave complicação da doença. Esse fato contribui para que esses indivíduos tenham a compreensão do risco em que estão expostos, fazendo com que os utentes aflorem a necessidade das realizações das ações de autocuidado, através dessa consciência de risco [18].

Ainda nesse contexto, a percepção que o indivíduo tem sobre seu próprio corpo é uma maneira de poder auxilia-lo a reconhecer sua dor, angustias, esperanças e perspectivas e, desse modo, poder acrescentar novos conceitos na prática educativa em autocuidado [10].

A capacitação da realização do autocuidado nem sempre está interligada com a prática da mesma. Muitas vezes o sujeito possui competência para praticar o cuidado consigo mesmo em prol da sua própria saúde, mas pouco se é explorado sobre esse domínio, ou seja, o indivíduo que tem boa capacidade para o autocuidado não necessariamente o pratica, porém, conhecer sobre sua capacidade é uma vertente que pode ser explorada para a adesão desse utente. 
Nesse sentido, o estudo limita-se apenas à avaliação da capacidade, não considerando as práticas e suas realizações em si, visto que os participantes podem apresentar boas ou ótimas competências, mas não concretizam a execução do autocuidado. Portanto, é necessário que outros estudos analisem tais práticas, suas frequências e qualidades.

Além disso, ressalta-se inviabilidade de acesso a alguns indivíduos em virtude de dificuldades estruturais, violência urbana e vulnerabilidade à pesquisadora. Neste contexto, fazse necessário a realização de novos estudos com a utilização da escala ASA-A para ampliação do debate e fortalecimento da importância da adesão ao autocuidado.

Conclusão

O fato de o indivíduo ser acometido pela hanseníase remete a uma avalanche de consequências negativas em relação a sua vida física, psíquica e sociocultural, provinda, principalmente, pelas incapacidades e deformidades físicas que acarretam junto a si o estigma, discriminação, preconceito, exclusões e desestruturação da imagem corporal, que podem ser evitadas pela aderência ao autocuidado.

Diante dos dados expostos, pode-se observar uma taxa majoritária dos entrevistados apresentando competência para se autocuidar. A mensuração dessa capacidade é importante para compreender a efetividade da realização do autocuidado e através deste poder construir uma melhor assistência integral ao paciente, respeitando sua individualidade.

Concernente aos demais achados neste estudo, pode-se perceber a interligação entre o sítio corporal facial, assim como a forma clínica predominante multibacilar, como fatores predispostos que se aproximam à adesão da prática do autocuidado.

Neste sentido, é necessária a realização de treinamentos/capacitações dos profissionais de saúde de forma continuada para subsidiar o tratamento e a prevenção de incapacidades através do autocuidado, levando em consideração as particularidades, limitações e aspectos culturais, assim como o potencial de cada indivíduo para exercer o autocuidado.

1. World Health Organization. Weekly epidemiological record 2018;93(35):445-56.

2. Talhari C, Talhari S, Penna GO. Clinical aspects of leprosy. Clin Dermatol 2015;33(1):26-37. https://doi.org/10.1016/j.clindermatol.2014.07.002

3. Gaudenci EM, Nardelli GG, Almeida Neto OP, Malaquias BSS Carvalho BT, Pedrosa LAK. Qualidade de vida, sintomas depressivos e incapacidade física de pacientes com hanseníase. Hansen Int 2015;40(2):48-58.

4. Ministério da Saúde (BR). Tecnologia da informação a serviço do SUS. Situação epidemiológica hanseníase Brasil. Brasília: Ministério da Saúde; 2018.

5. Oliveira LR, Nascimento AR, Nascimento MMP, Pereira AP, Lemos ICS, Kerntopf MR. Limitação de atividades e participação social entre usuários de um grupo de autocuidado em hanseníase. Revista Interdisciplinar 2016;9(1):171-81.

6. Buna ATM, Rocha FDCG, Alves EM, Granja FBC, Sousa DJ, Silva MGP. Incapacidades físicas nos pacientes com hanseníase cadastrados em uma unidade de saúde de São Luís-MA. Revista Interdisciplinar 2016;8(1):115-22.

7. Brasil; Ministério da Saúde; Secretaria de Vigilância em Saúde; Departamento de Vigilância das Doenças Transmissíveis. Diretrizes para vigilância, atenção e eliminação da Hanseníase como problema de saúde pública: manual

8. técnico-operacional. Brasília: Ministério da Saúde; 2016.

9. Lima MCV, Barbosa FR, Santos DCM, Nascimento RD, D'Azevedo SSP. Practices for self-care in Hansen's disease: face, hands and feet. Rev Gaúcha Enferm 2018;39:e20180045. https://doi.org/10.1590/1983-1447.2018.20180045

10. Hernández YN, Pacheco JAC, Larreynaga MR. La teoría déficit de autocuidado: Dorothea Elizabeth Orem. Gac Méd Espirit 2017;19(3):1-13.

11. Batista TVG, Vieira CSCA, Paula, MAB. A imagem corporal nas ações educativas em autocuidado para pessoas que tiveram hanseníase. Physis 2014;24(1):89-104. https://doi.org/10.1590/S0103-73312014000100006

12. Sousa GS, Silva RLF, Xavier MB. Hanseníase e Atenção Primária à Saúde: uma avaliação de estrutura do programa. Saúde Debate 2017;41(112):230-42.

https://doi.org/10.1590/0103-1104201711219 
13. Ministério da Saúde (BR). Secretaria de Atenção à Saúde. Departamento de Atenção Básica. Política Nacional de Atenção Básica. Brasília: Ministério da Saúde; 2012.

14. Silva JV, Domingues EAR. Adaptação cultural e validação da escala para avaliar as capacidades de autocuidado. Arq Ciênc Saúde 2017;24(4):30-6.

15. Aquino CMF, Rocha EPAA, Guerra MCG, Coriolano MWL, Vasconcelos EMR, Alencar EN. Peregrinação (Via Crucis) até o diagnóstico da hanseníase. Rev Enferm UERJ 2015;23(2):185-90. https://doi.org/: 10.12957/reuerj.2015.12581

16. Mendes $E V$. O cuidado das condições crônicas na atenção primária à saúde. Rev Bras Promoç Saúde 2018;31(2):1-3. https://doi.org/ 10.5020/18061230.2018.7565

17. Albano ML, Sousa AAS, Cezário KG, Pennafort VPS, Américo CF. A consulta de enfermagem no contexto de cuidado do paciente com hanseníase. Rev Hansen Int 2016;41(1-2):25-33.

18. Silva JSR, Palmeira IP, Sá AMM, Nogueira LMV, Ferreira AMR. Variáveis clínicas associadas ao grau de incapacidade física na hanseníase. Rev Cuid 2019;10(1):e618. https://doi.org/10.15649/cuidarte.v10i1.618

19. Ericeira VVL. Hanseníase: avaliação das limitações das atividades, consciência de risco e participação social [Dissertação]. São Luís (MA): Universidade Federal do Maranhão; 2017. 94 f. 